\title{
A model for quasi-spherical magnetized accretion flow
}

\begin{abstract}
M. Shadmehri
Department of Physics, School of Science, Ferdowsi University, Mashhad, Iran

e-mail: mshadmehri@science1.um.ac.ir

Received 29 March 2004 / Accepted 11 May 2004

Abstract. A model for axisymmetric magnetized accretion flow is proposed. The dominant mechanism of energy dissipation is assumed to be the magnetic diffusivity due to turbulence in the accretion flow. In analogy to the advection-dominated accretion flow (ADAF) solutions, a constant fraction of the resistively dissipated energy is stored in the accreting gas and the rest is radiated. We first introduce the general self-similar solutions which describe a resistive and nonrotating flow with purely poloidal magnetic field. The radial dependence of physical quantities is identical to that in viscous ADAF solutions. Although the main focus of this study is on nonrotating magnetized accretion flow, for rotating flow with both poloidal and toroidal components of magnetic field we find a radial scaling of solutions similar to the nonrotating case. We show that the accretion and the rotation velocities are both below the Keplerian rate, irrespective of the amount of cooling. We show that the set of equations is reduced to one second order differential equation for a nonrotating flow. The geometrical shape of the disk changes depending on the fraction of the resistively dissipated energy which is stored in the accreting gas. However, there is a hot low-density gas above the disk in almost all cases. The net accretion rate is calculated for a set of illustrative parameters.
\end{abstract}

Key words. accretion, accretion disks - magnetohydrodynamics (MHD)

\section{Introduction}

The importance of the accretion process has been recognized in connection with the physics of many astrophysical systems such as protosteller disks (e.g., Mineshige \& Umemura 1997; Tsuribe 1999; Calvet et al. 2000), disks around black holes or compact objects, or disks at the center of active galactic nuclei (e.g., Lynden-Bell \& Rees 1971; Kato et al. 1998). According to their geometrical shapes, accretion disks are generally divided into thin and thick disks. A mechanism for the transport of angular momentum is another key ingredient in the theory of accretion processes and many theoretical uncertainties remain about its nature. However, the theory of thin disks is well understood based on a pioneer work by Shakura \& Sunyaev (1973). In this theory it is assumed that there is efficient radiation cooling in the flow.

During recent years another type of accretion disk has been studied, in which the energy released through viscous processes in the disk may be trapped within the accreting gas (see, e.g., Ichimaru 1977; Narayan \& Yi 1995, hereafter NY). In this kind of flow which is known as advection-dominated accretion flow (ADAF), the accreting gas has a very low density and is unable to cool efficiently. A full analysis of the dynamics of the solution was presented in an important paper by NY. Since both the angular momentum and the energy dissipation in the flow is due to the turbulent viscosity as in the standard $\alpha$ model, we can call such flows "viscous" ADAF models.
There is growing evidence that the accretion flows in many astrophysical systems involve magnetized plasma. Accretion disks containing magnetic fields have been studied by many authors (e.g., Ogilvie 1997; Hawley 2001; Casse \& Keppens 2002). However, the standard viscous ADAF model completely ignores the presence of an ordered global magnetic field and it is assumed that the turbulent magnetic field contributes a constant fraction of the total pressure (NY). However, we can expect accretion flows, in which angular momentum is transported by a global magnetic field and the energy is released by Joule heating. This possibility has been addressed by Kaburaki (2000). He presented a set of analytical solutions for a fully advective accretion flow in a global magnetic field and the conductivity is assumed to be constant for simplicity.

Schwartzman (1971) was the first to point out the importance of the magnetic field in an accretion process. He proposed a hypothesis of equipartition between the magnetic and kinetic energy densities and this picture as mentioned above is usually accepted in the modern picture of viscous ADAF models (e.g., NY). A more accurate study was done by BisnovatyiKogan \& Ruzmaikin (1974), where an exact nonstationary solution for field amplification in the radial accretion flow was also obtained. Also, Bisnovatyi-Kogan \& Lovelace (2000) suggested that recent papers discussing ADAF as a possible solution for astrophysical accretion should be treated with caution, particularly because of ignorance of the magnetic field. While they obtained a solution for a time-averaged magnetic field in a 
quasispherical accretion flow, an analysis of energy dissipation and equipartition between magnetic and flow energies has been presented (Bisnovatyi-Kogan \& Lovelace 2000). In our study, we follow similar ideas in order to study magnetized accretion flow in which magnetic energy dissipation is stored in the flow.

The dynamics of radiatively inefficient accretion flows has been the subject of many studies during recent years (e.g., Igumenshchev \& Abramowicz 1999, 2000; Stone et al. 1999; McKinney \& Gammie 2002). In these viscous accretion models, the behaviour of the flow depends on the standard dimensionless viscosity parameter $\alpha$. On the other hand, as rotation is allowed, such flows become subject to strong convection with completely different flow pattern and radiative properties (Igumenshchev \& Abramowicz 1999; Stone et al. 1999). Numerical MHD simulations of radiatively inefficient flows have been done recently by many authors (e.g., Hawley 2001; Machida et al. 2001; Casse \& Keppens 2002). However, in most of these the resistive terms in the MHD equations have been neglected, or the resistivity has been considered only in the induction equation without accounting the corresponding dissipation in the energy equation.

In this paper, we want to explore how the structure of a steady-state thick disk depends on its resistivity by solving the two-dimensional MHD equations for disks which have the simplifying feature that they are self-similar in radius. We will consider the general problem of accretion flow where the field annihilation is approximated by a finite "turbulent" conductivity and the viscosity of the fluid is completely neglected in order to construct a fully resistive model for accretion within a global magnetic field. We restrict our attention to a nonrotating accretion flow which contains a purely poloidal magnetic field. We will discuss the general case in a future paper. However, we will find the radial scaling of self-similar solutions for the general case where the fluid is rotating and contains both poloidal and toroidal components of the magnetic field.

This paper is organized as follows. In Sect. 2 the general problem of constructing a model for resistively accretion flow is defined. The self-similar solutions are presented in Sect. 3, and the effects of the input parameters are examined. The implications of this analysis are summarized in Sect. 4.

\section{Formulation of the problem}

As stated in the introduction, we are interested in constructing a model for describing magnetized accretion disks. The macroscopic behavior of such flows can be studied by MHD approximation. For simplicity, the self-gravity and general relativistic effects have been neglected. The first assumption is true as long as the total mass of the disk is small compared with the product of the mass of central object and the angular thickness of the disk. Thus, the basic equations of our problem are written as follows:

$$
\begin{aligned}
& \frac{\partial \rho}{\partial t}+\nabla \cdot(\rho \boldsymbol{v})=0, \\
& \rho\left(\frac{\partial \boldsymbol{v}}{\partial t}+(\boldsymbol{v} \cdot \nabla) \boldsymbol{v}\right)=-\nabla p-\rho \nabla \Psi+\frac{1}{4 \pi} \boldsymbol{J} \times \boldsymbol{B},
\end{aligned}
$$

$$
\begin{aligned}
& \frac{\partial \boldsymbol{B}}{\partial t}=\nabla \times(\boldsymbol{v} \times \boldsymbol{B}-\eta \boldsymbol{J}), \\
& \frac{\rho}{\gamma-1} \frac{\mathrm{d}}{\mathrm{d} t}\left(\frac{p}{\rho}\right)+p \nabla . \boldsymbol{v}=Q_{\mathrm{diss}}-Q_{\mathrm{cool}}
\end{aligned}
$$

$\nabla . B=0$,

where $\rho, v, p, \Psi$ are the density, the velocity, the pressure and the gravitational potential due to a central object, respectively. We assume that the mass of the central object is $M$, and so the gravitational potential becomes $\Psi=-G M / r$ where $r$ is the spherical radial coordinate. Also, $\boldsymbol{J}=\nabla \times \boldsymbol{B}$ is the current density and $\eta$ represents the magnetic diffusivity. The term on the right hand side of the energy equation, $Q_{\text {diss }}$, is the rate of heating of the gas the dissipation, $Q_{\text {cool }}$ represents the energy loss through radiative cooling, and $\gamma$ is the adiapatic index. We assume that the difference between energy dissipation and radiative cooling is a fraction $f$ of energy dissipation, i.e. $Q_{\text {diss }}-Q_{\text {cool }}=f Q_{\text {diss }}($ e.g., $\mathrm{NY})$. The parameter $f$ measures the degree to which the flow is advection-dominated and in general it depends on the details of the heating and cooling mechanisms and will vary with both $r$ and $\theta$. However, we assume a constant $f$ for simplicity. Clearly, the case $f=1$ corresponds to the extreme limit of no radiative cooling, and in the limit of efficient cooling we have $f=0$.

We assume that the disk is stationary and axisymmetric. There are numerous studies in which magnetized equilibrium structures have been investigated via analytical or numerical methods (e.g., Igumenshchev \& Abramowicz 1999, 2000; Stone et al. 1999; Shalybkov \& Rüdiger 2000; Ogilvie \& Livio 2001; McKinney \& Gammie 2002; Rüdiger \& Shalybkov 2002). This paper will be restricted to nonrotating accretion flow in a purely poloidal magnetic fields configuration. The whole magnetic field is maintained by the electric current induced in the accretion region, and the solutions are determined only by the assumption of self-similarity. According to the antidynamo theorem (e.g., Cowling 1981), such a configuration cannot be maintained in a stationary state by an axisymmetric flow in the presence of resistive dissipation. We can meet this criticisms in two forms: for many problems the magnetic dissipation time is very long, much longer than the age of the system. For such problems, one assumes the field to have been generated in the distant past, and restricts one's attention to its effect on current dynamics, equilibrium, stability, etc.

For other problems the dissipation time is not longer than the age of the system. In such cases (as in this study), one is making some implicit assumption about the regeneration of the field by (dynamo) processes outside the computational regime of the problem at hand (e.g., on a 3D time-dependent microscale, or beyond the boundaries of the formal computation). Whether these assumptions are consistent with the computational details depends, of course, on the quality of the the author's physical intuition, since such assumptions lie beyond the physical and mathematical equations that are actually posed and solved.

There many studies in which the time-averaged physical quantities have been analyzed so that one can still talk about a stationary state, and this approach has been widely used in 
the literature (e.g., Lovelace et al. 1987, 1994; Shalybkov \& Rüdiger 2000; Ogilvie \& Livio 2001; Rüdiger \& Shalybkov 2002). In this study the physical quantities such as velocity $\boldsymbol{v}$, magnetic field $\boldsymbol{B}$ etc. denote the time-averaged values and in general, the instantaneous value of each physical variable consists of a stationary part and a fluctuating component. The influence of the fluctuating parts is included crudely, by modifying the transport coefficients which would apply in the absence of fluctuations. Thus, we neglect the time average of the fluctuating parts of velocity and magnetic field, and the generation of a magnetic field in the disk by dynamo activity is not included (e.g., Pudritz 1981). Since we are interested in nonrotating quasispherical accretion, one may ask about the instantaneous magnetic field lines. Recently, Bisnovatyi-Kogan \& Lovelace (2000) proposed a model for magnetized quasispherical accretion, in which a sketch of the instantaneous poloidal magnetic field lines and the time-averaged flow velocity has been shown in Fig. 1 of their work. As for the accretion flow within a global poloidal magnetic field, we are assuming the same configuration.

We work in spherical polar coordinates $(r, \theta, \varphi)$ with the origin on the central object. We assume that the net mass accretion rate $\dot{M}=-\int 2 \pi r^{2} \sin \theta \rho v_{\mathrm{r}} \mathrm{d} \theta$ is independent of radius $r$. Since $r^{2} \rho v_{\mathrm{r}}$ is independent of $r$ and the disk is stationary and axisymmetric, the continuity Eq. (1) shows $v_{\theta}=0$ and takes the form

$\frac{1}{r^{2}} \frac{\partial}{\partial r}\left(\rho v_{\mathrm{r}} r^{2}\right)=0$.

The condition of constant $\dot{M}$ is similar to what has been used by NY; however, it has been relaxed by some authors and they find solutions with outflows (e.g., Henriksen \& Valls-Gabaud 1994). In this paper, we consider the simple case of constant net mass accretion rate at all radii. We can write the components of the equation of motion (Eq. (2)) in spherical coordinates:

$\rho\left(v_{\mathrm{r}} \frac{\partial v_{\mathrm{r}}}{\partial r}-\frac{v_{\varphi}^{2}}{r}\right)=-\frac{\partial p}{\partial r}-\rho \frac{G M}{r^{2}}+\frac{1}{4 \pi}\left(J_{\theta} B_{\varphi}-J_{\varphi} B_{\theta}\right)$,

$-\rho v_{\varphi}^{2} \cot \theta=-\frac{\partial p}{\partial \theta}+\frac{r}{4 \pi}\left(J_{\varphi} B_{\mathrm{r}}-J_{\mathrm{r}} B_{\varphi}\right)$,

$\rho v_{\mathrm{r}} \frac{\partial v_{\varphi}}{\partial r}+\rho \frac{v_{\mathrm{r}} v_{\varphi}}{r}=\frac{1}{4 \pi}\left(J_{\mathrm{r}} B_{\theta}-J_{\theta} B_{\mathrm{r}}\right)$.

Also, the three components of induction Eq. (3) are:

$\frac{\partial}{\partial \theta}\left[r \sin \theta\left(v_{\mathrm{r}} B_{\theta}-\eta J_{\varphi}\right)\right]=0$,

$\frac{\partial}{\partial r}\left[r \sin \theta\left(v_{\mathrm{r}} B_{\theta}-\eta J_{\varphi}\right)\right]=0$,

$\frac{\partial}{\partial r}\left(r v_{\varphi} B_{\mathrm{r}}-r v_{\mathrm{r}} B_{\varphi}\right)+\frac{\partial}{\partial \theta}\left(v_{\varphi} B_{\theta}\right)-\frac{\partial}{\partial r}\left(\eta r J_{\theta}\right)+\frac{\partial}{\partial \theta}\left(\eta J_{\mathrm{r}}\right)=0$.

In Eqs. (10)-(12), the terms without $\eta$ represent the advection of the magnetic field by the flow motion, while the terms containing $\eta$ account for the diffusion of the field.
From Eqs. (10) and (11) one can simply deduce that $r \sin \theta\left(v_{\mathrm{r}} B_{\theta}-\eta J_{\varphi}\right)=0$, or

$v_{\mathrm{r}} B_{\theta}-\eta J_{\varphi}=0$.

This important equation can be understood easily in this way: In a stationary, axisymmetric configuration, the electric field can be written as the gradient of a scalar potential, and its $\phi$ component (i.e., $E_{\varphi}$ ) which is in proportion to $v_{\mathrm{r}} B_{\theta}-\eta J_{\varphi}$ in our notation, should vanish identically. This expression appears in the parentheses in Eqs. (10) and (11). So, these equations reduce into one equation, i.e. Eq. (13), as we showed above. We will find self-similar solutions that satisfy Eq. (13).

The energy Eq. (4) and the magnetic flux conservation give

$\rho\left(\frac{v_{\mathrm{r}}}{\gamma-1} \frac{\partial}{\partial r}\left(\frac{p}{\rho}\right)-\frac{p}{\rho^{2}} v_{\mathrm{r}} \frac{\partial \rho}{\partial r}\right)=f Q_{\mathrm{diss}}$,

$\frac{1}{r^{2}} \frac{\partial}{\partial r}\left(r^{2} B_{\mathrm{r}}\right)+\frac{1}{r \sin \theta} \frac{\partial}{\partial \theta}\left(B_{\theta} \sin \theta\right)=0$,

where in the above equations the components of the current density $\boldsymbol{J}$ are

$$
\begin{aligned}
& J_{\mathrm{r}}=\frac{1}{r \sin \theta} \frac{\partial}{\partial \theta}\left(B_{\varphi} \sin \theta\right), \quad J_{\theta}=-\frac{1}{r} \frac{\partial}{\partial r}\left(r B_{\varphi}\right), \\
& J_{\varphi}=\frac{1}{r}\left[\frac{\partial}{\partial r}\left(r B_{\theta}\right)-\frac{\partial B_{\mathrm{r}}}{\partial \theta}\right] .
\end{aligned}
$$

To close the system of equations, we should specify the functional forms of the magnetic diffusivity $\eta$ and the energy dissipation $Q_{\text {diss }}$. In this study, we have completely neglected the effects of viscous processes, and our attention has been restricted to resistive dissipation. In other words, the accretion flow of our model is not infinitely conducting. For simplicity, we could assume the resistivity to be constant (see, e.g., Kaburaki 2000). However, we assume that the magnetic diffusivity is due to turbulence in the accretion flow and it is reasonable to express this parameter in analogy to the $\alpha$-prescription of Shakura \& Sunyaev (1973) for the turbulent viscosity,

$\eta=\eta_{0} \frac{c_{\mathrm{s}}^{2}}{\Omega_{\mathrm{K}}}=\eta_{0} \frac{p}{\rho \Omega_{\mathrm{K}}}$,

where $\Omega_{\mathrm{K}}=\sqrt{G M / r^{3}}$ is the Keplerian angular velocity, and $c_{\mathrm{s}}$ is the isothermal sound speed. NY applied a similar functional form for kinematic coefficient of viscosity, i.e. $v=$ $\alpha\left(p / \rho \Omega_{\mathrm{K}}\right)$. Exactly in analogy to NY's prescription, we are using the above equation for the magnetic diffusivity $\eta$. Note that $\eta$ is not constant and depends on the physical variables of the flow, and in our self-similar solutions, as we will show, $\eta$ scales with radius as $r^{1 / 2}$. The magnetic diffusivity has the same units as kinematic viscosity and we assume that the magnitude of $\eta$ is comparable to that of the turbulent viscosity (Bisnovatyi-Kogan \& Ruzmaikin 1976; Parker 1979), although we have neglected kinematic viscosity. This form of scaling for diffusivity has been widely used by many authors (e.g., Lovelace et al. 1987; Lovelace et al. 1994; Ogilvie \& Livio 2001; Rüdiger \& Shalybkov 2002). Due to the arbitrary $\eta_{0}$ it is not so important which velocity is used for the scaling. 
For example, in some studies Alfvén velocity is used for the velocity in the above scaling (e.g., Fendt \& Cemeljic 2002), while in others the sound speed is still applied even in the magnetized case (e.g., Lovelace et al. 1994; Rüdiger \& Shalybkov 2002). Nevertheless, the sound speed, it seems, has an advantage; because it is the largest intrinsic velocity parameter in the turbulent plasma, and may be used universally, with or without magnetic field. In this study, following many previous studies, we use the above parameterized form for the diffusivity.

For the heating term, $Q_{\text {diss }}$, we may have two sources of dissipation. Magnetic reconnection may lead to energy release. Also, we can consider the viscous and resistive dissipations due to a turbulence cascade. In this study, only resistive dissipation has been considered as the dominant process: $Q_{\mathrm{diss}}=\frac{\eta}{4 \pi} J^{2}$.

Now we have constructed our model and the main equations of the model are Eqs. (6)-(9) and (12)-(15). In the next section, we will present self-similar solutions of these equations.

\section{Analysis}

\subsection{Self-similar solutions}

To better understand the physics of our accretion flow in a global magnetic field we seek self-similar solutions of the above equations. Of course, this method is familiar from its wide applications to the full set of equations of MHD in many research fields of astrophysics. As long as we are not interested in the boundaries of the problem, such solutions that describe the behavior of the flow in an intermediate region far from the radial boundaries.

Before presenting the self-similar solutions, we introduce a convenient functional form for the magnetic field. Generally, the later may have both poloidal and toroidal components. We can define a positive-definite magnetic flux function $\Phi(r, \theta)$ from which we may obtain the poloidal components of the magnetic field:

$\boldsymbol{B}=\frac{1}{2 \pi} \nabla \times\left(\frac{\Phi}{r \sin \theta} \boldsymbol{e}_{\varphi}\right)+B_{\varphi} \boldsymbol{e}_{\varphi}$

We can easily show that by integrating the above equation over the circular area $\pi r^{2}$ in the plane of $\theta=\pi / 2$, the result, i.e. $\Phi(r, \pi / 2)$, is the defined magnetic flux. This equation automatically satisfies Eq. (15).

Writing the equations in a non-dimensional form, that is, scaling all the physical variables by their typical values, brings out the non-dimensional variables. It is simple to show that a solution of the following form satisfies the equations of our model:

$$
\begin{aligned}
& \rho(r, \theta)=\rho_{0} \frac{R(\theta)}{\left(r / r_{0}\right)^{3 / 2}}, \\
& p(r, \theta)=p_{0} \frac{P(\theta)}{\left(r / r_{0}\right)^{5 / 2}}, \\
& v_{\mathrm{r}}(r, \theta)=r \Omega_{\mathrm{K}}(r) V(\theta), \\
& v_{\varphi}(r, \theta)=r \Omega_{\mathrm{K}}(r) \Omega(\theta),
\end{aligned}
$$

$$
\begin{aligned}
& B_{\mathrm{r}}(r, \theta)=\frac{B_{0}}{2 \pi \sin \theta} \frac{d \phi(\theta)}{d \theta} \frac{1}{\left(r / r_{0}\right)^{5 / 4}} \\
& B_{\theta}(r, \theta)=-B_{0} \frac{3 \phi(\theta)}{8 \pi \sin \theta} \frac{1}{\left(r / r_{0}\right)^{5 / 4}} \\
& B_{\varphi}(r, \theta)=B_{0} \frac{b(\theta)}{\left(r / r_{0}\right)^{5 / 4}}
\end{aligned}
$$

where $\rho_{0}, p_{0}, B_{0}$ and $r_{0}$ provide convenient units with which the equations can be written in non-dimensional form and $\Omega_{\mathrm{K}}$ denotes the Keplerian angular velocity, viz. $\Omega_{\mathrm{K}}(r)=\sqrt{G M / r^{3}}$. Also, we obtain the following equations of the components of current density:

$J_{\mathrm{r}}(r, \theta)=\frac{B_{0}}{r_{0}} \frac{1}{\sin \theta} \frac{\mathrm{d}}{\mathrm{d} \theta}(b(\theta) \sin \theta) \frac{1}{\left(r / r_{0}\right)^{9 / 4}}$,

$J_{\theta}(r, \theta)=\frac{B_{0}}{4 r_{0}} \frac{b(\theta)}{\left(r / r_{0}\right)^{9 / 4}}$

$J_{\varphi}(r, \theta)=\frac{B_{0}}{2 \pi r_{0}}\left[\frac{3 \phi(\theta)}{16 \sin \theta}-\frac{\mathrm{d}}{\mathrm{d} \theta}\left(\frac{1}{\sin \theta} \frac{\mathrm{d} \phi(\theta)}{\mathrm{d} \theta}\right)\right] \frac{1}{\left(r / r_{0}\right)^{9 / 4}}$

and considering Eq. (16), the magnetic diffusivity $\eta$ becomes

$\eta=\eta_{0} \frac{p_{0}}{\rho_{0} \sqrt{G M / r_{0}^{3}}} \frac{P(\theta)}{R(\theta)}\left(\frac{r}{r_{0}}\right)^{1 / 2}$.

Substituting the above solution in the momentum, induction and energy equations, we obtain a set of coupled ordinary differential equations. Note that the above solutions automatically satisfy continuity Eq. (6). Equations of motion (7), (8) and (9) become

$$
\begin{aligned}
R\left(-\frac{V^{2}}{2}-\right. & \left.\Omega^{2}\right)=\frac{5}{2} A_{1} P-R+\frac{A_{2}}{16 \pi} \\
& \times\left\{b^{2}+\frac{3 \phi}{4 \pi^{2} \sin \theta}\left[\frac{3 \phi}{16 \sin \theta}-\frac{\mathrm{d}}{\mathrm{d} \theta}\left(\frac{1}{\sin \theta} \frac{\mathrm{d} \phi}{\mathrm{d} \theta}\right)\right]\right\},
\end{aligned}
$$

$$
\begin{array}{r}
-R \Omega^{2} \cot \theta=-A_{1} \frac{\mathrm{d} P}{\mathrm{~d} \theta}+\frac{A_{2}}{4 \pi}\left\{\frac{1}{4 \pi^{2} \sin \theta} \frac{\mathrm{d} \phi}{\mathrm{d} \theta}\right. \\
\left.\times\left[\frac{3 \phi}{16 \sin \theta}-\frac{\mathrm{d}}{\mathrm{d} \theta}\left(\frac{1}{\sin \theta} \frac{\mathrm{d} \phi}{\mathrm{d} \theta}\right)\right]-\frac{b}{\sin \theta} \frac{\mathrm{d}}{\mathrm{d} \theta}(b \sin \theta)\right\}, \\
R V \Omega=-\frac{A_{2}}{4 \pi^{2} \sin \theta}\left[b \frac{\mathrm{d} \phi}{\mathrm{d} \theta}+\frac{3 \phi}{4 \sin \theta} \frac{\mathrm{d}}{\mathrm{d} \theta}(b \sin \theta)\right] .
\end{array}
$$

Equation (12) gives

$$
\begin{aligned}
2 \pi V b-\frac{\mathrm{d}}{\mathrm{d} \theta}(\Omega \phi)-\frac{\Omega}{\sin \theta} \frac{\mathrm{d} \phi}{\mathrm{d} \theta} & \\
+ & \frac{\eta_{0}}{2} \frac{P b}{R}+\frac{8 \pi \eta_{0}}{3} \frac{\mathrm{d}}{\mathrm{d} \theta}\left[\frac{P}{R \sin \theta} \frac{\mathrm{d}}{\mathrm{d} \theta}(b \sin \theta)\right]=0,
\end{aligned}
$$

and Eq. (13) is cast into this form

$\frac{\mathrm{d}}{\mathrm{d} \theta}\left(\frac{1}{\sin \theta} \frac{\mathrm{d} \phi}{\mathrm{d} \theta}\right)-\frac{3 \phi}{16 \sin \theta}-\frac{3}{4 \eta_{0} A_{1}} \frac{R V \phi}{P \sin \theta}=0$, 
and finally the energy Eq. (14) becomes

$$
\begin{aligned}
R V \frac{3 \gamma-5}{2(\gamma-1)}= & \frac{f \eta_{0} A_{2}}{4 \pi}\left\{\frac{1}{4 \pi^{2}}\left[\frac{3 \phi}{16 \sin \theta}-\frac{\mathrm{d}}{\mathrm{d} \theta}\left(\frac{1}{\sin \theta} \frac{\mathrm{d} \phi}{\mathrm{d} \theta}\right)\right]^{2}\right. \\
& \left.+\frac{b^{2}}{16}+\left[\frac{1}{\sin \theta} \frac{\mathrm{d}}{\mathrm{d} \theta}(b \sin \theta)\right]^{2}\right\},
\end{aligned}
$$

where

$$
\begin{aligned}
& A_{1}=\frac{p_{0}}{\rho_{0}\left(G M / r_{0}\right)}=\left(\frac{c_{0 \mathrm{~s}}}{v_{0 \mathrm{~K}}}\right)^{2}, \\
& A_{2}=\frac{B_{0}^{2}}{\rho_{0}\left(G M / r_{0}\right)}=4 \pi\left(\frac{v_{0 \mathrm{~A}}}{v_{0 \mathrm{~K}}}\right)^{2},
\end{aligned}
$$

where $v_{0 \mathrm{~K}}=\left(G M / r_{0}\right)^{1 / 2}$ is the Keplerian velocity, and $v_{0 \mathrm{~A}}=$ $B_{0} / \sqrt{4 \pi \rho_{0}}$ is the Alfvén velocity.

We can define the ratio of the thermal to the magnetic pressures $\beta$ as

$$
\beta(\theta)=\beta_{0} \frac{4 \pi^{2} P \sin ^{2} \theta}{9 \phi^{2} / 16+(\mathrm{d} \phi / \mathrm{d} \theta)^{2}+4 \pi^{2} b^{2} \sin ^{2} \theta},
$$

where $\beta_{0}=\frac{p_{0}}{B_{0}^{2} / 8 \pi}=8 \pi \frac{A_{1}}{A_{2}}$. Equations (29)-(34) constitute a system of ordinary non-linear differential equations for the six similarity variables $\phi(\theta), b(\theta), R(\theta), P(\theta), V(\theta)$ and $\Omega(\theta)$. Indeed, the behaviour of the solution depends on the boundary conditions for which assumptions are made based on some physical assumptions such as symmetry with respect to the equatorial plane. Ogilvie (1997) studied the equilibrium of magnetized non-accreting disks by assuming self-similarity in the spherical radial coordinate. If we neglect resistivity, our solution reduces to Ogilvie's solution, i.e. the model describes a non-accreting disk containing poloidal and toroidal components of the magnetic field. However, in the case of resistive accretion flow, the scalings of the quantities with spherical radius are still similar to those presented by Ogilvie (1997) and other authors (e.g., Blandford \& Payne 1982), i.e. $\rho \propto r^{-3 / 2}$, $p \propto r^{-5 / 2}$ and $\boldsymbol{B} \propto r^{-5 / 4}$.

The main topic of this study is nonrotating accretion flow. But an interesting feature of the solutions is worth emphasizing. Using Eq. (33), we can rewrite Eq. (29) as

$R\left(1-\frac{V^{2}}{2}-\Omega^{2}\right)=\frac{5}{2} A_{1} P+\frac{A_{2}}{16 \pi}\left(b^{2}-\frac{9 R V \phi^{2}}{16 \pi^{2} \eta_{0} P \sin ^{2} \theta}\right)$.

Since all the similarity variables are positive except for $V$, we see that the right hand side of Eq. (38) is positive for all $\theta$ except $\theta=0$ which is not evident from the equation. If we substitute Eqs. (33) and (34) into the last term of the right hand side of the above equation, a non-rotating flow within a purely poloidal field (i.e., $\Omega=0$ and $b=0$ ) which is our concern in this paper, is positively well-defined for all $\theta$, even in the limit of vanishing $\theta$. However, if we consider both the toroidal and the poloidal components of the magnetic field, we can still say that when $\theta$ tends to zero, the last term of the right hand side of Eq. (38) even if it tends to a very large value, would be negative (because $V$ is negative, the rest of the variables are positive) and so the right hand side of the equation is positive (there is a minus sign before the last term of the right hand side). Thus we should have

$$
\frac{V^{2}}{2}+\Omega^{2} \leq 1
$$

This inequality implies rotation velocities below the Keplerian rate for the solutions of the model, irrespective of the amount of cooling or value of resistivity $\eta_{0}$. Also, we can say that, as the value of the angular velocity increases, the accretion velocity decreases. In other words, in this model the high accretion rate corresponds to a low rotation rate. In this study, we shall restrict our attention to a non-rotating accretion flow, i.e. $\Omega=0$.

\subsection{Nonrotating accretion solutions}

What we have is a set of complicated differential equations which must be solved under appropriate boundary conditions. Although a full numerical solution of Eqs. (29)-(34) would now be possible, it is more instructive to proceed by analyzing the model in some restrictive conditions such as non-rotation. To derive this solution we set $\Omega=0$ and $b=0$; the latter condition means that we consider purely poloidal magnetic fields. However, to further simplify the problem, we cannot impose spherical symmetry, i.e. $\mathrm{d} / \mathrm{d} \theta \equiv 0$. Because if we impose spherical symmetry, we can not identify the actual magnetic field lines, nor can we account for nonradial forces. But we know that in resistive accretion flow the topology of the magnetic field plays a vital role.

Like NY we define the parameters $\epsilon=\frac{5 / 3-\gamma}{\gamma-1}$ and $\epsilon_{\mathrm{f}}=\frac{\epsilon}{f}$. As we will see the parameter $\epsilon_{\mathrm{f}}$ plays an important role in the behaviour of the solutions. Obviously, when we set $\Omega=0$ and $b=0$, Eqs. (31) and (32) immediately drop out, and Eqs. (29), (30) and (34) can be written as

$$
\begin{aligned}
& R\left(1-\frac{V^{2}}{2}\right)= \frac{5}{2} A_{1} P-\frac{3 A_{2}}{64 \pi^{3}} \\
& \times \frac{\phi}{\sin \theta}\left[\frac{\mathrm{d}}{\mathrm{d} \theta}\left(\frac{1}{\sin \theta} \frac{\mathrm{d} \phi}{\mathrm{d} \theta}\right)-\frac{3 \phi}{16 \sin \theta}\right] \\
& A_{1} \frac{\mathrm{d} P}{\mathrm{~d} \theta}+\frac{A_{2}}{16 \pi^{3} \sin \theta}\left[\frac{\mathrm{d}}{\mathrm{d} \theta}\left(\frac{1}{\sin \theta} \frac{\mathrm{d} \phi}{\mathrm{d} \theta}\right)-\frac{3 \phi}{16 \sin \theta}\right] \frac{\mathrm{d} \phi}{\mathrm{d} \theta}=0 \\
& {\left[\frac{\mathrm{d}}{\mathrm{d} \theta}\left(\frac{1}{\sin \theta} \frac{\mathrm{d} \phi}{\mathrm{d} \theta}\right)-\frac{3 \phi}{16 \sin \theta}\right]^{2}+\frac{24 \pi^{3} \epsilon_{\mathrm{f}}}{\eta_{0} A_{2}} R V=0 }
\end{aligned}
$$

and Eq. (33) remains unchanged. Now, the set of equations reduces to four Eqs. (33), (40), (41) and (42) which we want to solve.

Clearly the expressions inside the brackets in Eqs. (40), (41) and (42) are similar, and by substituting from Eq. (33), these three equations become

$R\left(1-\frac{V^{2}}{2}\right)=\frac{5}{2} A_{1} P-\frac{9 A_{2}}{256 \pi^{3} \eta_{0} A_{1}} \frac{R V \phi^{2}}{P \sin ^{2} \theta}$

$A_{1} \frac{\mathrm{d} P}{\mathrm{~d} \theta}+\frac{3 A_{2}}{64 \pi^{3} \eta_{0} A_{1}} \frac{R V \phi}{P \sin ^{2} \theta} \frac{\mathrm{d} \phi}{\mathrm{d} \theta}=0$ 
$\frac{R V \phi^{2}}{P^{2} \sin ^{2} \theta}+\frac{128 \pi^{3} \eta_{0} \epsilon_{\mathrm{f}} A_{1}^{2}}{3 A_{2}}=0$.

From Eq. (45), we obtain

$R V=-\frac{128 \pi^{3} \eta_{0} \epsilon_{\mathrm{f}} A_{1}^{2}}{3 A_{2}} \frac{P^{2} \sin ^{2} \theta}{\phi^{2}}$,

and by substituting $R V$ from the above equation into Eqs. (43) and (44), we have

$R\left(1-\frac{V^{2}}{2}\right)=\left(\frac{5+3 \epsilon_{\mathrm{f}}}{2}\right) A_{1} P$,

$\frac{1}{P} \frac{\mathrm{d} P}{\mathrm{~d} \theta}+\frac{2 \epsilon_{\mathrm{f}}}{\phi} \frac{\mathrm{d} \phi}{\mathrm{d} \theta}=0$.

Fortunately, Eq. (48) is integrable and gives

$P=\phi^{n}$,

where $n=2 \epsilon_{\mathrm{f}}$. Now, considering the above result, we can rewrite Eqs. (46) and (47) as

$$
\begin{gathered}
R V=-\frac{128 \pi^{3} \eta_{0} \epsilon_{\mathrm{f}} A_{1}^{2}}{3 A_{2}} \phi^{2 n-2} \sin ^{2} \theta, \\
R\left(1-\frac{V^{2}}{2}\right)=\left(\frac{5+3 \epsilon_{\mathrm{f}}}{2}\right) A_{1} \phi^{n} .
\end{gathered}
$$

From these two equations, one can easily calculate $V$ and $R$ as functions of $\phi$ as follows

$$
V(\theta)=\frac{1-\sqrt{1+2 a^{2} \phi^{2(n-2)} \sin ^{4} \theta}}{a \phi^{n-2} \sin ^{2} \theta},
$$

where

$a=\frac{32 \pi^{2} \beta_{0} \eta_{0} \epsilon_{\mathrm{f}}}{3\left(5+3 \epsilon_{\mathrm{f}}\right)}$,

and by substituting $V$ as a function of $\phi$ into Eqs. (50) or (51), we can find $R$ as a function of $\phi$. Thus, if we can find $\phi$, all physical variables are known. Equation (33) is the last equation which gives us $\phi$ as a function of $\theta$. Considering Eqs. (49) and (50), we can rewrite Eq. (33) as

$\frac{\mathrm{d}}{\mathrm{d} \theta}\left(\frac{1}{\sin \theta} \frac{\mathrm{d} \phi}{\mathrm{d} \theta}\right)-\frac{3 \phi}{16 \sin \theta}+4 \pi^{2} \beta_{0} \epsilon_{\mathrm{f}} \phi^{n-1} \sin \theta=0$

or,

$\frac{\mathrm{d}^{2} \phi}{\mathrm{d} \xi^{2}}-\frac{3 \phi}{16\left(1-\xi^{2}\right)}+4 \pi^{2} \beta_{0} \epsilon_{\mathrm{f}} \phi^{n-1}=0$,

where $\xi=\cos \theta$. Now we have a second order ordinary differential equation for $\phi(\theta)$ which can be solved using a numerical method (in the Appendix we give an analytical solution for $n=1$ ). One boundary condition can be specified in the equatorial plane, by assuming that the field lines thread the equator perpendicularly:

$\left(\frac{\mathrm{d} \phi}{\mathrm{d} \theta}\right)_{\theta=\pi / 2}=0$
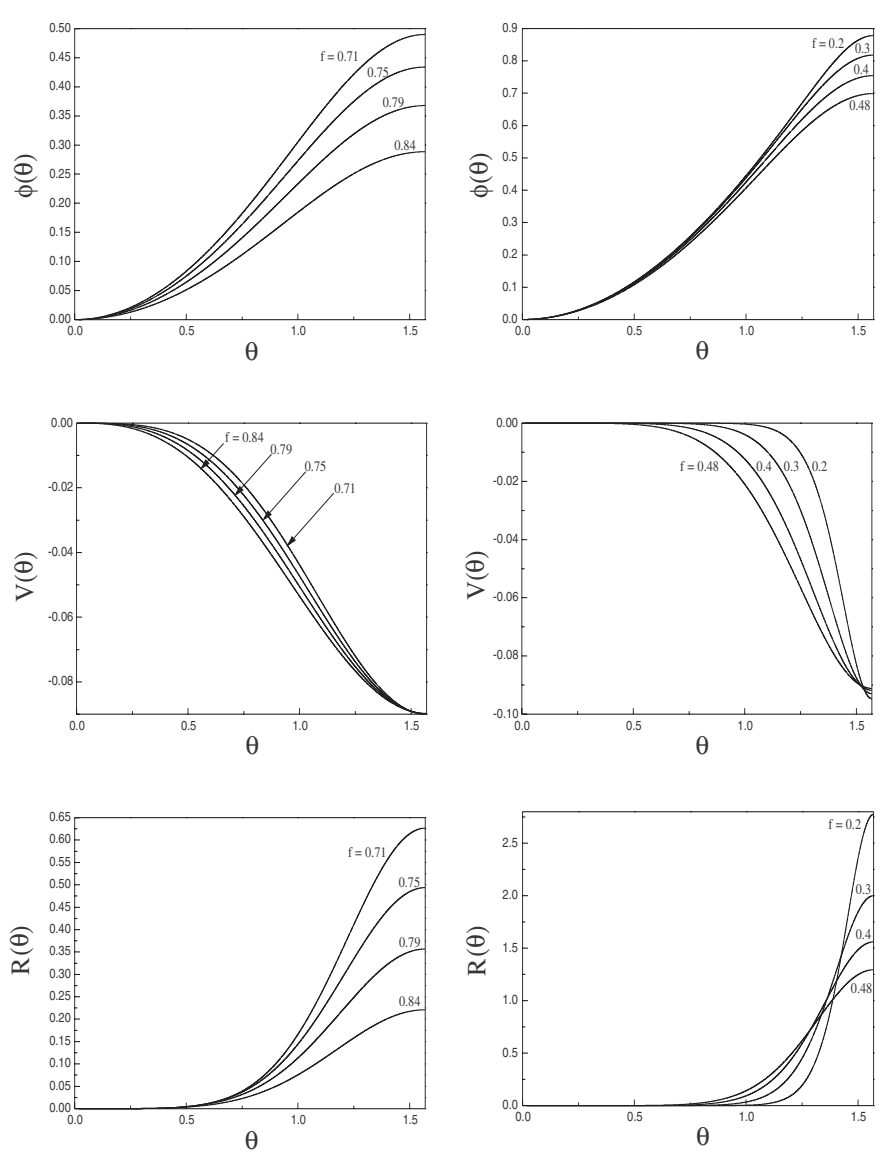

Fig. 1. Self-similar solutions corresponding to $\gamma=4 / 3, \eta_{0}=0.1$ and $\beta_{0}=0.1$. Top: magnetic flux function $\phi(\theta)$ as a function of polar angle $\theta$. Middle: Radial velocity $V(\theta)$. Bottom: density $R(\theta)$.

The second boundary condition can be specified by demanding that the magnetic flux enclosed by a very small cone around the polar axis vanishes:

$\phi(0)=0$.

Thus, the complicated set of differential equations is reduced to a boundary value problem of a second order differential equation. We have obtained numerical solutions of the above equation for different values of $f$ and $\eta_{0}$. For our illustrative parameters, we assume $r_{0}=1 \mathrm{pc}, B_{0}=10^{-5} \mathrm{G}, M=10^{6} M_{\odot}, \beta_{0}=0.1$ and $\dot{M}_{0}=2 \pi \rho_{0} r_{0}^{2} \sqrt{G M / r_{0}}=1.2 \times 10^{-4} M_{\odot} /$ year which gives $\rho_{0}=2 \times 10^{-20} \mathrm{~g} / \mathrm{cm}^{3}$. Figure 1 shows some particular solutions corresponding to $\eta_{0}=0.1, \gamma=4 / 3$ and different values of the advected energy $f$. For a fixed value of $\gamma$, the solutions imply increasing cooling. This general behavior is the same as in NY for viscous ADAF.

As the six panels in Fig. 1 show, the behaviour of the solutions, is very sensitive to the value of $f$ or $\epsilon_{\mathrm{f}}$. The top panels show the variations of the magnetic flux function $\phi(\theta)$ as a function of $\theta$. While this similarity function of the magnetic flux is zero on the polar axis (this is a boundary condition), it has a maximum value on the equator which increases with decreasing $f$. This growing flux contrast between the equatorial region and the polar region implies that at a fixed radius on the equator, the magnetic flux increases with decreasing $\gamma$ (or, equivalently, increasing cooling). 
The middle panels show the similarity function of the radial velocity $V(\theta)$ (see Eq. (52)). We see that the maximum accretion velocity is in the equatorial region, and on the polar axis there is no mass inflow. As we expected, the accretion velocity is sub-Keplerian. Although for our representative set of parameters the variation of $V(\theta)$ as a function of $\theta$ decreases from equatorial to polar region, we can recognize two distinct regions in the general profile of $V(\theta)$ depending on the value of the velocity. The bulk of the accretion occurs between the equatorial plane at $\theta=\pi / 2$ and a surface at $\theta=\theta_{\mathrm{f}}$, inside of which the velocity $V(\theta)$ is zero. Note that all of our solutions have well-behaved non-sigular $V(\theta)$ at all $\theta$, and the radial velocity does not diverge at $\theta=\theta_{\mathrm{f}}$. Also, for the flows which extend from the equatorial plane to a surface at $\theta=\theta_{\mathrm{s}}$ where $\theta_{\mathrm{f}}<\theta_{\mathrm{s}}<\pi / 2$, the accretion velocity is decreasing. With decreasing $f$, the region with maximum accretion velocity becomes narrow, i.e. $\theta_{\mathrm{s}}$ decreases.

In the bottom panels of Fig. 1, we plot the density similarity function $R(\theta)$. The density contrast between equatorial and polar regions increases with decreasing $f$. Like for the magnetic flux function $\phi(\theta)$, as $f$ decreases the density grows and becomes concentrated toward the equatorial plane. As already mentioned, the small $f$ solutions correspond to efficient cooling for a fixed $\gamma$. Thus the $R(\theta)$ profile clearly shows what we expect with increasing cooling, or equivalently $\epsilon_{\mathrm{f}}$. More interestingly, although the solutions for large $\epsilon_{\mathrm{f}}$ imply that the bulk of the accretion occurs in the equatorial region (i.e., like a thin disk configuration), in all cases there is a low density with higher temperature above the disk as the bottom panels of Fig. 1 show. While the bulk of the accreting gas along the equatorial plane has approximately constant temperature, this tenuous region also has a constant higher temperature. As the cooling increases, the temperatures of both regions decrease. Note that in the tenuous region there is no accretion.

From these figures we see for fixed values of $\gamma$ and $\eta_{0}$ that, as the advected energy decreases and more energy radiates out of the system, the magnetic flux function $\phi(\theta)$ increases. It means that magnetic field lines can penetrate the disk more easily when we have cooling. Thus, while the thermal pressure decreases due to the cooling, the magnetic pressure increases and the ratio of these two becomes lower which is important in analyzing the dynamics of the flow. All solutions correspond to the poloidal magnetic field configuration, in which the field lines bend only once when passing through the equatorial plane.

Figure 2 shows this ratio of the thermal to the magnetic pressure. We see that this ratio decreases as the amount of the advected energy $f$ decreases. Figure 3 shows the magnetic field lines for a typical self-similar solution with $\eta_{0}=$ 0.1 and $f=0.84$. For other input parameters we can obtain the same configuration. Ogilvie (1997) studied a nonaccreting magnetized disk by considering a polytropic equation of state. However, the magnetic field configuration in his study is similar to what has been obtained in this study for resistive accretion flow. If we neglect turbulent diffusivity and the energy equation, our solutions reduce to Ogilvie's solutions. However, in our scenario two main factors in the dynamics of the flow are the diffusion mechanism of the magnetic field lines, and the

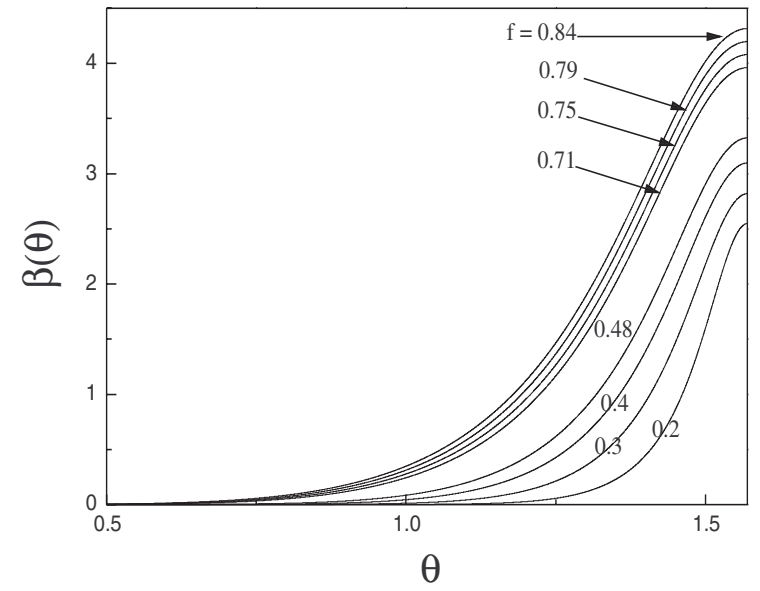

Fig. 2. Ratio of the thermal to the magnetic pressures for the solutions shown in Fig. 1.

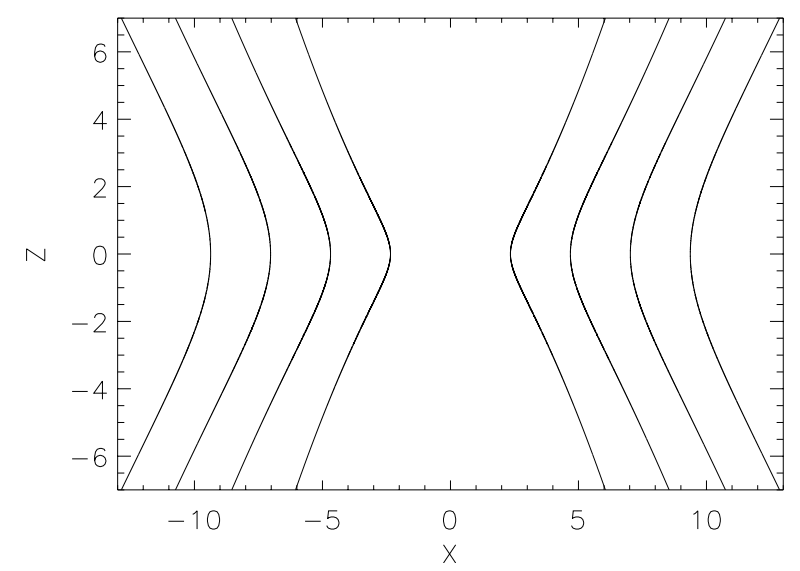

Fig. 3. Representation of magnetic field lines in the meridional plane for a typical self-similar solution with $\eta_{0}=0.1, \gamma=4 / 3$ and $f=0.84$.

advected energy. For example, the net rate of accretion depends on the enclosed flux function $\phi(\theta)$. We can write

$\dot{M}=-2 \dot{M}_{0} \int_{0}^{\frac{\pi}{2}} \sin \theta R(\theta) V(\theta) \mathrm{d} \theta$.

Considering Eqs. (42) and (49), we can rewrite the above equation as

$\dot{M}=\frac{16}{3} \pi^{2} \beta_{0} n \eta_{0} A_{1} \dot{M}_{0} \int_{0}^{\frac{\pi}{2}} \phi^{2 n-2} \sin ^{3} \theta \mathrm{d} \theta$.

Although this kind of magnetized accretion flow is different from Bondi (1952) accretion in various aspects, we can define the Bondi accretion rate for the illustrative parameters as $\dot{M}_{\text {Bondi }} \simeq 4 \pi G^{2} M^{2} \rho_{0} / c_{\mathrm{s}}^{3}$. Thus, $\dot{M}_{\text {Bondi }}=2\left(v_{0 \mathrm{~K}} / c_{0 \mathrm{~s}}\right)^{3} \dot{M}_{0}$. Assuming $A_{1}=1$, we can write the net accretion rate as follows

$\dot{M}=\frac{8}{3} \pi^{2} \beta_{0} n \eta_{0} \dot{M}_{\text {Bondi }} \int_{0}^{\frac{\pi}{2}} \phi^{2 n-2} \sin ^{3} \theta \mathrm{d} \theta$.

For the flows which we have at hand, the mass accretion rate is at below the Bondi accretion rate for a wide range of input parameters (e.g., $\epsilon_{\mathrm{f}}$ and $\eta_{0}$ ). Figure 4 shows the net mass accretion rate $\dot{M} / \dot{M}_{\text {Bondi }}$ as a function of the advected energy $f$ for 


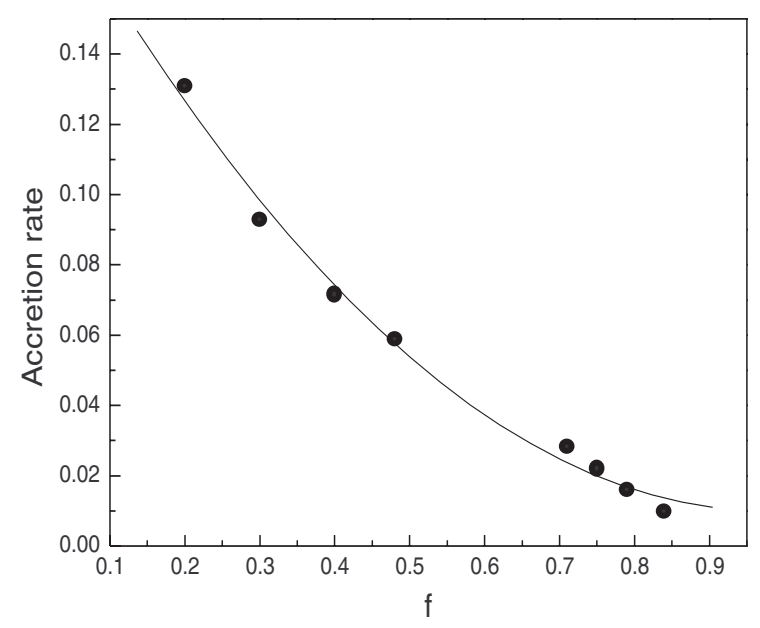

Fig. 4. The net accretion rate $\dot{M} / \dot{M}_{\text {Bondi }}$ as a function of the advected energy $f$ for the self-similar solutions shown in Fig. 1.

$\gamma=4 / 3$ and $\eta_{0}=0.1$. As the above equation shows, the mass accretion rate changes in proportion to $\eta_{0}$. For these parameters, we have fitted an approximate function, viz. $\dot{M} / \dot{M}_{\text {Bondi }} \approx$ $0.19-0.38 f+0.19 f^{2}$. The radial structure of the resistive radiatively inefficient accretion flow is dramatically modified from the Bondi profile, and the mass accretion rate decreases significantly if the advected energy $f$ is increased. We see that, for a fixed value of $\eta_{0}$, the enclosed flux function $\phi(\theta)$ increases as $\epsilon_{\mathrm{f}}$ increases (i.e., cooling increases) and the net mass accretion rate increases as well. Thus, in our model the accretion rate is always suppressed compared to the Bondi rate, although the precise amount depends on the cooling and the resistivity. There are many numerical simulations which show an infall of non-rotating or slowly-rotating magnetized gas with reduced $\dot{M}$ (e.g., Igumenshchev et al. 2000; Hawley et al. 2001). However, our simple model for magnetized accretion flow shows this behavior clearly.

\section{Discussion and conclusions}

In this paper we have obtained axisymmetric magnetized selfsimilar advection-dominated flow solutions by considering an ordered magnetic field and resistive dissipation of the energy of the flow. The radial dependence of the solutions has the same behaviour as that of ADAF solutions (NY), but the angular parts are obtained by solving a set of ordinary differential equations. In the model, the viscosity of the flow is completely neglected in order to make the contrast between the standard viscous and resistive ADAF solutions clear. We showed that the radial and the rotational velocities are well below the Keplerian velocity, and this is independent of the resistivity and the amount of cooling. However, the main aim of this study was to study the nonrotating quasi-spherical magnetized flow directly just by solving the relevant MHD equations. The same problem has been addressed by many authors, under the assumption that the field will achieve equipartition of magnetic and kinetic energies (see, e.g., Shvartsman 1971; NY; Bisnovatyi-Kogan \& Lovelace 2000). Bisnovatyi-Kogan \& Lovelace (2000) discussed equipartition between magnetic and kinetic energies using time-averaged magnetic field solution. They argued that with such an equipartition half of the dissipated energy of the accretion flow results from the destruction of the magnetic field. In this paper we showed that the equipartition depends on the input parameters, more importantly the amount of advected energy $\left(\epsilon_{\mathrm{f}}\right)$ and the resistivity $\eta_{0}$. Also, the non-magnetized problem is described by pure hydrodynamics; it was solved by Bondi (1952) and has been widely applied. Note that our solutions differ in various aspects from Bondi's (1952) spherical accretion. While the magnetic field diffusivity is the main accretion factor in accretion in our model, the energy equation has been considered as well. In Bondi accretion, there is no mechanism for energy transport. Note that in pure Bondi spherical flow, self-similar accretion is allowed only for a single value of $\gamma$. However, a magnetized, rediatively inefficient accretion flow is possible for a range of values of $\gamma$. The other interesting feature is that the dynamics of such a flow depends not only on the magnetic diffusivity $\eta_{0}$, but also on the fraction of advected energy $f$.

For a nonrotating resistive accretion flow, the system of equations simplified to a second order differential equation. For given $\epsilon_{\mathrm{f}}$ and $\eta_{0}$ this equation determines the magnetic field, and the other physical variables can be found using the set of algebraic equations we obtained. Although we have neglected ingredients such as angular momentum and viscosity, the general properties of our solutions are similar to ADAF solutions. More importantly, the geometrical shape of the flow is determined by the amount of the advected energy (i.e., $\epsilon_{\mathrm{f}}$ ) for a given $\eta_{0}$. While solutions with efficient cooling resemble thin disks, the radiatively inefficient accretion describes nearly quasi-spherical flow.

The bulk of accretion with nearly constant velocity occurs in a region which extends from the equatorial plane to a surface at $\theta_{\mathrm{s}}$, inside of which the accretion velocity gradually decreases to zero. Thus, the accretion velocity is constant in the region $\pi / 2<\theta<\theta_{\mathrm{s}}$. As $\epsilon_{\mathrm{f}}$ decreases, the size of this region increases and the accretion tends to a quasi-spherical flow. The temperature of this region is also constant and is surrounded by a region with low density and higher temperature. This hot gas corona is similar to what has been discussed in viscous ADAF solutions (NY). As the cooling increases and the flow tends to a disk-like configuration, the size of this corona increases.

The ratio of the thermal to the magnetic pressure $\beta(\theta)$ depends on the input parameters $\epsilon_{\mathrm{f}}$ and $\eta_{0}$. In the limit of large $\epsilon_{\mathrm{f}}$, $\beta(\theta)<1$ for all $\theta$. This means that the thermal pressure is not in equipartition with the magnetic pressure, and, more precisely, the magnetic pressure is stronger than the thermal pressure in this regime. On the other hand, for inefficient cooling flows we found that the value of the ratio exceeds one for a range of $\theta$. Still, there is no equipartition between thermal and magnetic pressure except for a surface at a specific angle which can be determined.

As for the rate of heating $Q_{\text {diss }}$, resistive dissipation at small scales as a result of a turbulence cascade has been assumed to be the dominant mechanism. However, there are other sources, such as energy release through magnetic reconnection, and, more importantly, viscous dissipation. Phenomenological considerations may lead us to define $Q_{\text {diss }}$ as a fraction of the 
gravitational potential energy of the accreting gas. This simple definition allows one to consider all the dissipation processes without having detailed models for them. Preliminary results for this form of $Q_{\text {diss }}$ show that the scaling of quantities with radius still remains similar to our model, but the other properties of the solutions should be studied.

Since we completely neglected viscous dissipation, the turbulent magnetic Prandtel number of our model is zero. This unsatisfactory aspect can be removed by considering the viscosity and the resistivity simultaneously. Fortunately, the radial scaling of the physical variables in the resistive case, as we showed, is similar to the viscous case. By dimensional analysis, one can show in a simple way that self-similarity is possible as long as $\eta$ (and viscosity $v$ ) scales with radius as $r^{1 / 2}$. Thus it is still possible to find self-similar solutions in the general case. Given the importance of the value of the turbulent magnetic Prandtel number we can construct a model for viscous-resistive accretion flow in which this number is a free parameter.

\section{Appendix A:}

Equation (55) can be solved analytically for $n=1$. Assuming

$\phi(\xi)=\frac{16}{35} 4 \pi^{2} \beta_{0} \epsilon_{\mathrm{f}}\left(1-\xi^{2}\right)+y(\xi)$,

Eq. (55) reduces to

$\frac{\mathrm{d}^{2} y}{\mathrm{~d} \xi^{2}}-\frac{3}{16} \frac{y}{1-\xi^{2}}=0$.

By redefining the variables as

$z=\frac{1+\xi}{2}, y=z Y$

this equation becomes

$z(1-z) \frac{\mathrm{d}^{2} Y}{\mathrm{~d} z^{2}}+(2-2 z) \frac{\mathrm{d} Y}{\mathrm{~d} z}-\frac{3}{16} Y=0$.

The general solution of this hypergeometric equation is

$Y(z)=C_{1} F\left(\frac{3}{4}, \frac{1}{4}|2| z\right)+C_{2} F\left(\frac{3}{4}, \frac{1}{4}|2| z\right) \int \frac{\mathrm{d} z}{\left[z F\left(\frac{3}{4}, \frac{1}{4}|2| z\right)\right]^{2}}$,

where $C_{1}$ and $C_{2}$ are arbitrary constants and $F$ is the hypergeometric function.

\section{References}

Bisnovatyi-Kogan, G. S., \& Ruzmaikin, A. A. 1976, Ap\&SS, 42, 401 Bisnovatyi-Kogan, G. S., \& Lovelace, R. V. E. 2000, ApJ, 529, 978

Blandford, R. D., \& Payne, D. G. 1982, MNRAS, 199, 883

Bondi, H. 1952, MNRAS, 112, 195

Calvet, N., Hartmann, L., \& Strom, S. E. 2000, in Protostars and Planets IV, ed. V. Mannings, A. P. Boss, \& S. S. Russell (Tucson: University of Arizona Press), 377

Cowling, T. G. 1981, ARA\&A, 19, 115

Casse, F., \& Keppens, R. 2002, ApJ, 581, 988

Fendt, C., \& Cemeljic, M. 2002, A\&A, 395, 1045

Hawley, J. F. 2001, ApJ, 554, 534

Hawley, J. F., Balbus, S. A., \& Stone, J. M. 2001, ApJ, 554, L49

Henriksen, R. N., \& Valls-Gabaud, D. 1994, MNRAS, 266, 681

Ichimaru, S. 1977, ApJ, 214, 840

Igumenshchev, I. V., \& Abramowicz, M. A. 1999, MNRAS, 303, 309

Igumenshchev, I. V., \& Abramowicz, M. A. 2000, ApJS, 130, 463

Igumenshchev, I. V., Abramowicz, M. A., \& Narayan, R. 2000, ApJ, 537, L27

Kaburaki, O. 2000, ApJ, 531, 210

Kato, S., Fukue, J., \& Mineshige, S. 1998, Black-hole accretion disks (Kyoto: Kyoto University Press)

Lovelace, R. V. E., Wang, J. C. L., \& Sulkanen, M. E. 1987, ApJ, 315, 504

Lovelace, R. V. E., Romanova, M. M., \& Newman, W. I. 1994, ApJ, 437, 136

Lynden-Bell, D., \& Rees, M. J. 1971, MNRAS, 152, 461

Machida, M., Matsumoto, R., \& Mineshige, S. 2001, PASJ, 53, L1

McKinney, J. C., \& Gammie, C. F. 2002, ApJ, 573, 728

Mineshige, S., \& Umemura, M. 1997, ApJ, 480, 167

Narayan, R., \& Yi, I. 1995, ApJ, 444, 231 (NY)

Ogilvie, G. I. 1997, MNRAS, 288, 63

Ogilvie, G. I., \& Livio, M. 2001, ApJ, 553, 158

Parker, E. N. 1979, Cosmical magnetic fields (Oxford: Clarendon Press), Chap. 17

Pudritz, R. E. 1981, MNRAS, 195, 881

Rüdiger, G., \& Shalybkov, D. A. 2002, A\&A, 393, L81

Shakura, N. I., \& Sunyaev, R. A. 1973, A\&A, 24, 337

Shalybkov, D., \& Rüdiger, G. 2000, MNRAS, 315, 762

Shvartsman, V. F. 1971, SvA, 15, 377

Stone, J. M., Pringle, J. E., \& Begelman, M. C. 1999, MNRAS, 310, 1002

Tsuribe, T. 1999, ApJ, 527, 102 